\title{
RESEARCHING ON REAL 3D MODELING CONSTRUCTED WITH THE OBLIQUE PHOTOGRAMMETRY AND TERRESTRIAL PHOTOGRAMMETRY
}

\author{
Han Youmei ${ }^{1,2}$ Jiao Minglian $^{1}$ Shijuan $^{1}$ \\ ${ }^{1}$ Huaihai Institute of technology, Lian yungang, Jiangsu Province, China. \\ ${ }^{2}$ Beijing Key Laboratory of Urban Spatial Information Engineering, Beijing, China
}

KEYWORDS: Oblique photogrammetry; combined the oblique and the terrestrial photogrammetry; real 3D modeling; automatic modeling

\section{ABSTRACT:}

With the rapid development of the oblique photogrammetry, many cities have built some real 3D model with this technology. Although it has the advantages of short period, high efficiency and good air angle effect, the near ground view angle of these real 3D models are not very good. With increasing development of smart cities, the requirements of reality, practicality and accuracy on real 3D models are becoming higher. How to produce and improve the real 3D models quickly has become one of the hot research directions of geospatial information. To meet this requirement In this paper, Combined with the characteristics of current oblique photogrammetry modeling and the terrestrial photogrammetry, we proposed a new technological process, which consists of close range sensor design, data acquisition and processing. The proposed method is being tested by using oblique photography images acquired. The results confirm the effectiveness of the proposed approach.

\section{INTRODUCTION}

The oblique photography technology has been a new technology developed in recent years. With several cameras on one air platform, it can obtain the top and sides data of the buildings synchronously. This new technology has improved the limitation of the traditional photogrammetry because of the high overlapped and multi-view data (Yang Guodong, Wang Mingshui). The real 3D model constructed by this technology can meet some requirement of the smart city construction. Until now, it has been used for emergency command, homeland security, urban management etc.

Han Youmei, School of Geomatics and Marine Information, Huaihai Institute of Technology, Lian Yungang, Jiangsu, China. Email:hanyoumei@126.com , Funded by Beijing Key Laboratory of Urban Spatial Information Engineering No.2018213, and Key Laboratory of Precise Engineering and Industry Surveying, National Administration of Surveying, Mapping and Geoinformation No.PF2017-3
At present, the application of oblique photogrammetry technology has been widely carried out, and the real 3D model constructed by it has gradually become an important part of the urban spatial data framework. However, the near ground parts of the real 3D model constructed by ithas some deformation $(\mathrm{Qu}$ Lin etc), texture fuzzy, which can't meet the higher requirement of the smart city construction. We intended to improve the real 3D by combining the terrestrial photogrammetry data with the oblique photogrammetry.

\section{THE MODELING PROCESS}

\subsection{The design of the process}

The oblique photogrammetry is good at the top and some high sides real 3D model construction. According to the oblique photogrammetry principle we designed one new ground multi-view images acquisition device. By using this new device we acquire some ground and building sides images, after that, 
we combined the oblique photogrammetry images with the terrestrial data to construct the more perfect real 3D model, which is better in the geometry and the texture.

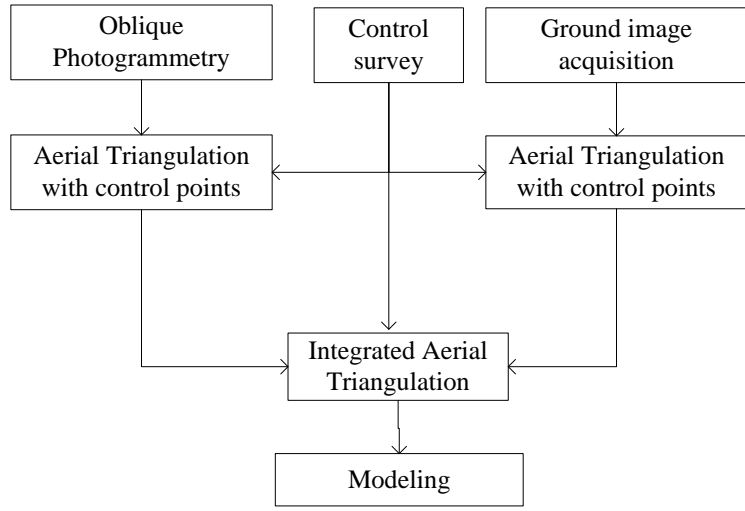

Figure 1 The integration modeling process flow chart

Because of the different angel and resolution of the air and terrestrial images, direct aerial triangulate (AT) with oblique photogrammetry data and the ground images is difficult. So in this paper, both the air images and terrestrial images need to operate AT respectively, after that, the two results above are combined to do AT again to get refine result, finally the refined result are used to model.(figure 1 )

\subsection{The key technologies}

\subsubsection{Terrestrial images acquire technology}

The traditional terrestrial images are generally acquired with one digital camera or digital camera with GPS. But these kinds of sensors always lead to more workload especially for the relative bigger projects. We designed a new kind of sensor to solve this problem. It consist of six different direct cameras and one GPS which on the top of it (Figure2). The adjacent cameras must have some deigned overlap. This new device can acquire data with some distance or time interval. Meanwhile the position of each image can be got by the GPS. So it can improve efficiency of data acquirement.

The idea of this design are following:

1) On one side it needs to cover all vertical direction images on one point, on the other hand it needs to keep cost;

2) The overlap of the adjacent cameras view should be not less than $30 \%$;

3) It can keep these six camera obtain images synchronously and can obtain data by some distance or some time intervals;
4) There is one GPS antenna on the top of the design which enable us to get the image position by it;

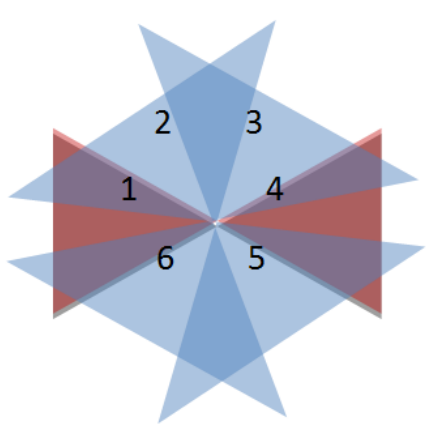

The design draft of the new sensor (a)

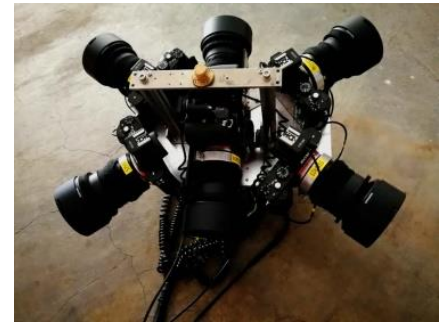

The physical equipment of the design

(b)

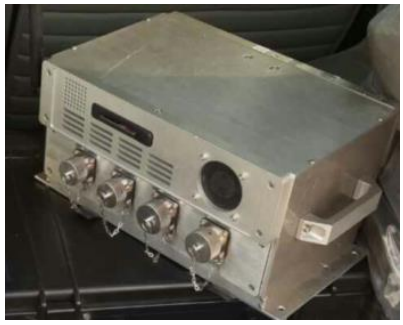

The control device of the new sensor (c)

Figure 2 The design and the physical figures of our new device

After some experiment, we chose six cameras to set up the new device. No.1 camera is defined as the left camera and No. 2 camera is defined as the left front camera. No.3 camera is defined as the right front camera and No. 4 camera is defined as the right camera. No.5 camera is the right back camera, and No.6 camera is defined as the left back camera. According to the photogrammetry principle, the overlap between two adjacent cameras is not less than $30 \%$. to acquire the modeling data, the new device is installed on the vehicle top. And the images overlap between the directions of the left or right camera is around $80 \%$, and a certain sampling interval is set to ensure this condition. 


\subsubsection{The control points layout scheme}

There are some papers about the oblique photogrammetry control point layout scheme (Hu wenqing, Zhou Xiaomin etc.), but in order to carry out the AT with the near ground and aerial images together, these schemes can't be used directly. So a new kind of control point layout scheme was proposed. There are at least four control points per square kilometer, most of the control points are on the ground and the road intersection. The most important is all of the control points should be seen from the aerial images and the terrestrial images.

\section{THE EXPERIMENT OF THESE NEW INVENTION}

\subsection{The overview of the experimental area}

The experimental area is a general park with many low and medium height buildings in it. The air condition is good at acquiring data by oblique photogrammetry. And the roads are very convenient for the terrestrial data acquisition. The area is about 1 square kilometers.

\subsection{Data acquisition}

The design ground resolution of oblique photogrammetry is about $2 \mathrm{~cm}$. The sensor for oblique photogrammetry is one aerial device named ARC 524(Figure.3) made by Shanghai Hang Yao information technology company, which consists of six cameras. The focal length of the downward camera is $20 \mathrm{~mm}$, and the focal length of the side view cameras is $35 \mathrm{~mm}$. The flight trajectory in experimentation area is figure 4 .
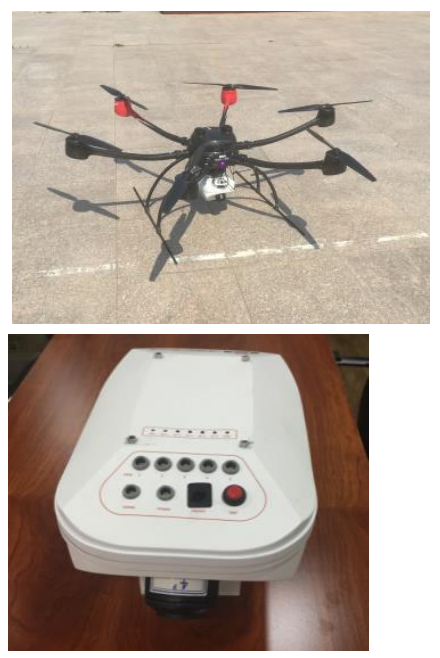

Figure 3 The Unmanned Aerial Vehicle and the aerial camera

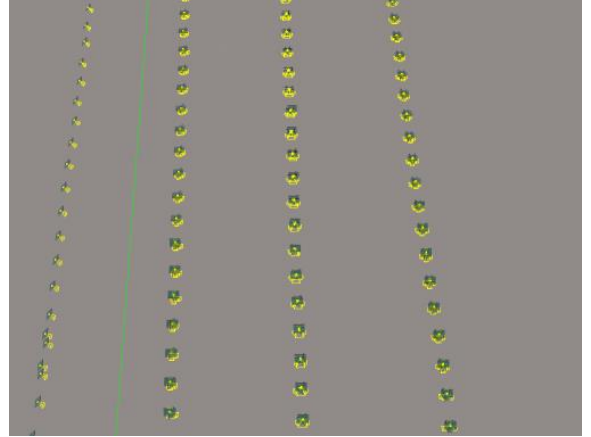

Figure 4 The flight trajectory in experimentation area

The terrestrial photogrammetry was acquired by the new device invented in this research. And the single camera is about 20 million pixels. The overlap of the terrestrial image is designed at $85 \%$. The specific acquisition interval was calculated by the following formula 1):

$$
\frac{f}{p}=\frac{d}{D}
$$

Where $f$ is the focal length of the left or the right camera,

$p$ is the width of the phase amplitude in the horizontal direction of one single camera, the $d$ is the width of the image,

$D$ is the estimated distance from the camera to the main facing object, such as the side of some high building, etc.. (1-85\%) $d$ is the sampling distance.

The trajectory of the terrestrial image collection in the experimentation area is as follow.

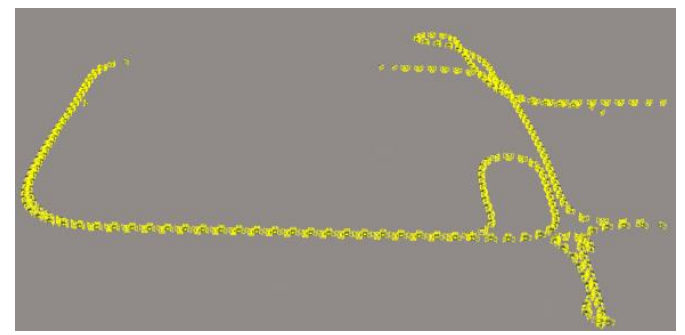

Figure 5 Trajectory of the near ground image acquisition

\subsection{The Control Survey}

The control measurement is carried out by network RTK (Figure 6), and three measurements are measured at each point, and the average value is taken as the final result.

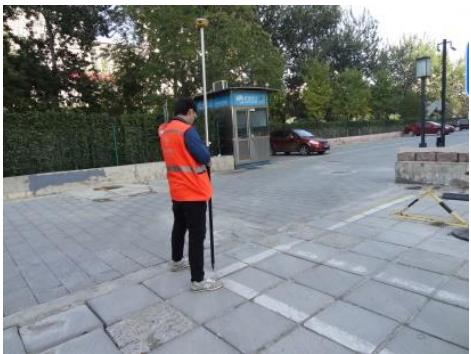


Figure 6 The Control points survey scene

\subsection{The data processing}

The data processing consists of image preprocessing, the input of position files of images, AT and the modeling. It is noted that the shared control points are added to carry out AT combined the aerial images with the ground images. In data processing, the software Context Capture (Context Capture User Manual version 4.4.) is used to carried out AT and modeling.

\subsection{Analysis of model effect}

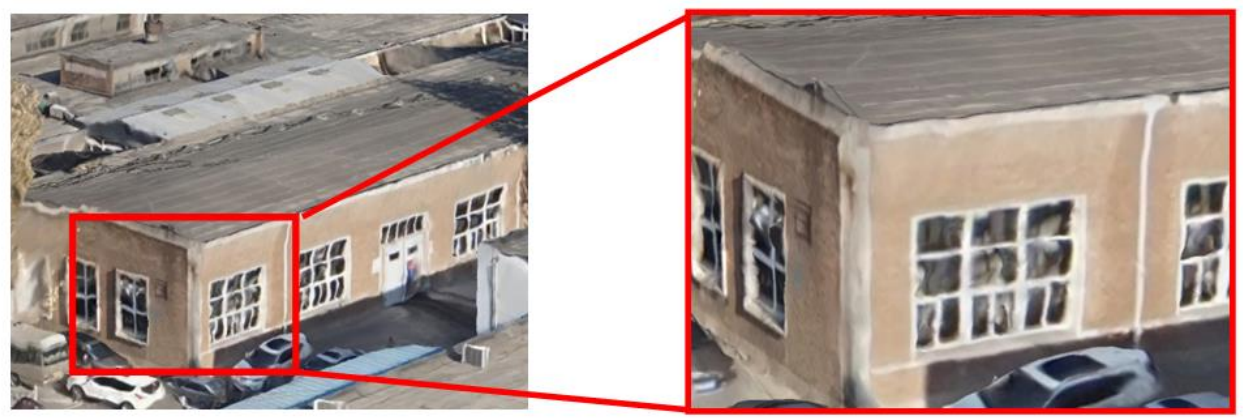

Figure 7 The model constructed only by the oblique photogrammetry

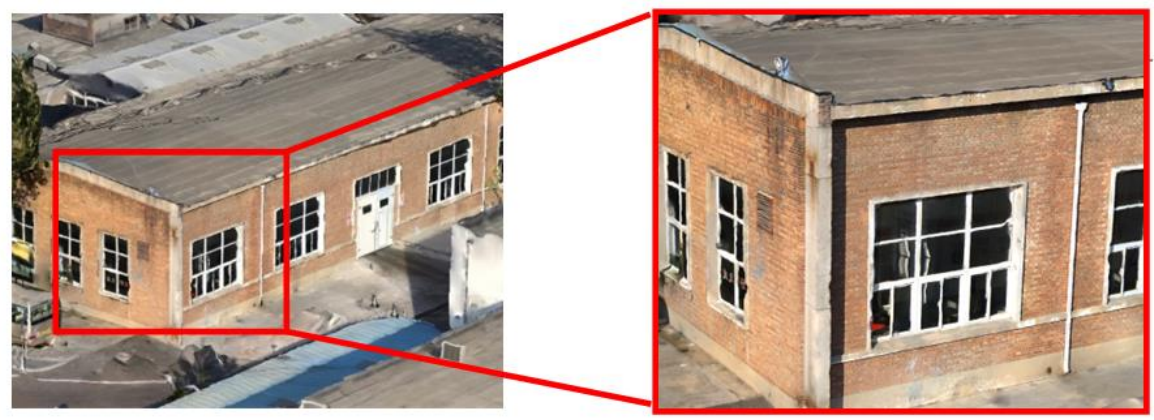

Figure 8 The model constructed by the oblique photogrammetry and the ground photogrammetry

In Figure 7 , the model produced by the oblique photogrammetry has some deformmation, such as near ground windows, roof edges, eaves etc.. After adding the terrestrial image data, the geometric information of the model is greatly improved.

It can be seen from partial enlargement image that texture information and the geometric information of the buildings have been greatly improved(figure 8 ). The main reason is that the terrestrial photos have high resolution and make up the ground view of the models. The model under the eaves has also been greatly improved compared with the result of produced by oblique photogrammetry only. And the big experiment area model are showed in figure 9 and figure 10. 


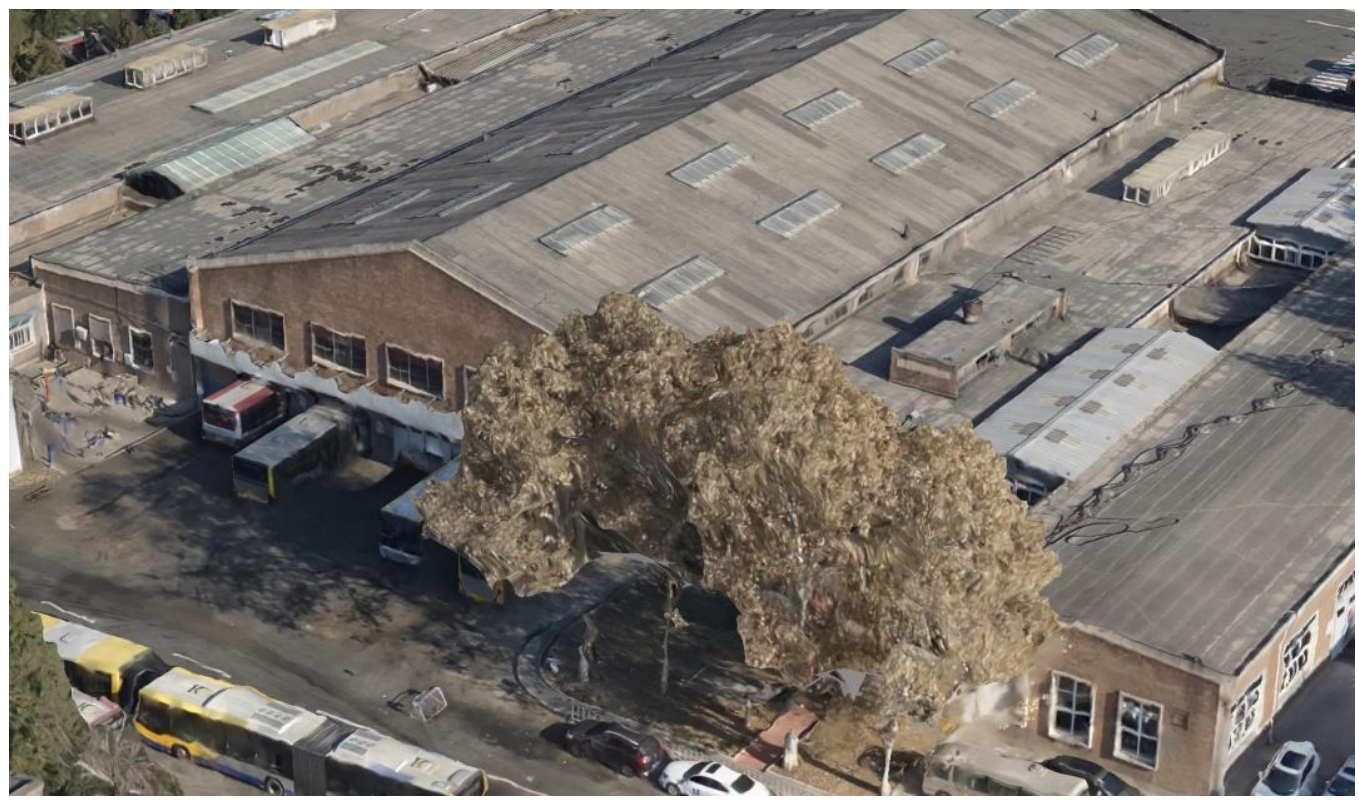

Figure 9 The big experiment area model produced by the oblique photogrammetry only

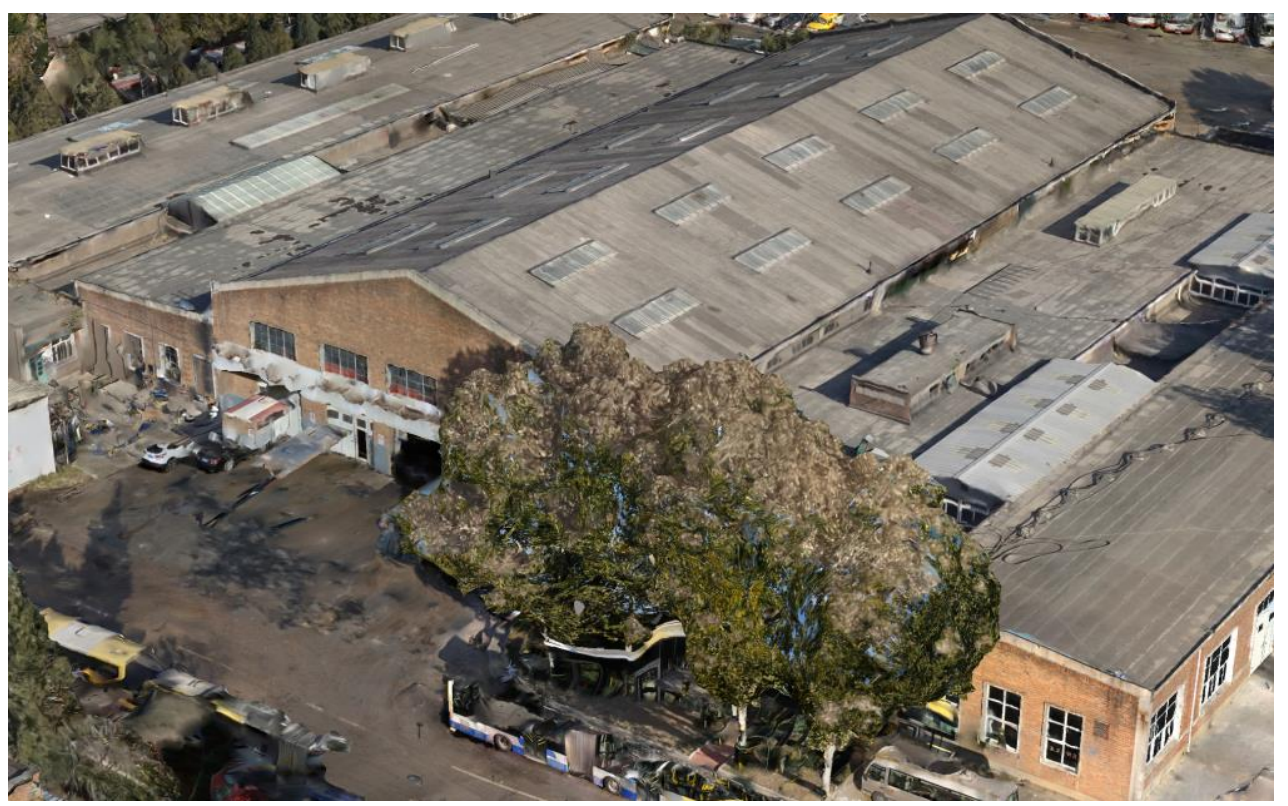

Figure 10 The big experiment area model produced by the oblique photogrammetry and the close range photogrammetry In order to verify the results of the experiment, this paper has another region, and the results are shown as follows: carried out the modeling experiments of the terrestrial image in
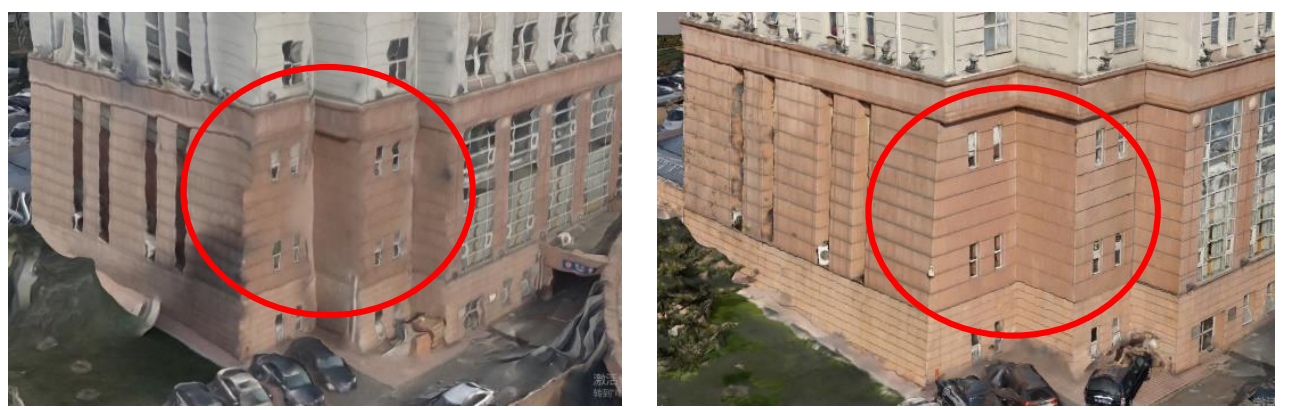

Figure. 11 The side of one building model produced by oblique photogrammetry $(\mathrm{OP})$ only(left) and produced by OP and 
terrestrial photogrammetry(right)

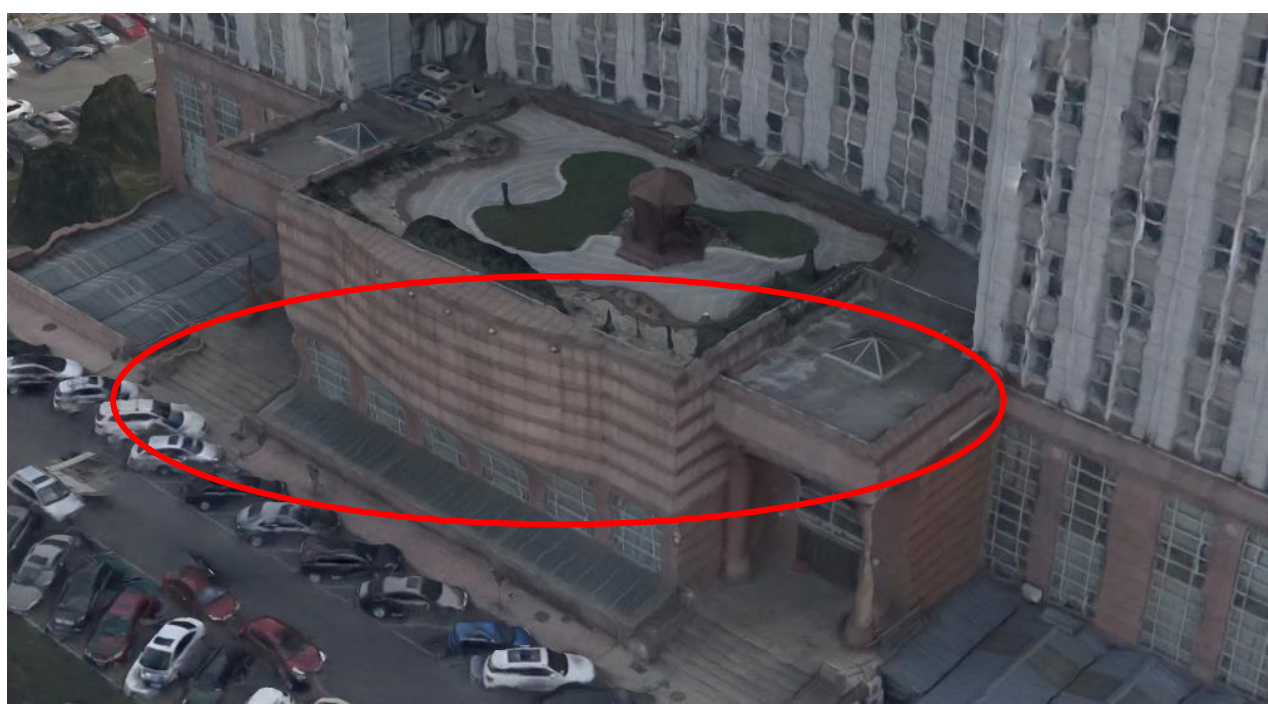

Figure 12 The air view of one big building model produced by OP only

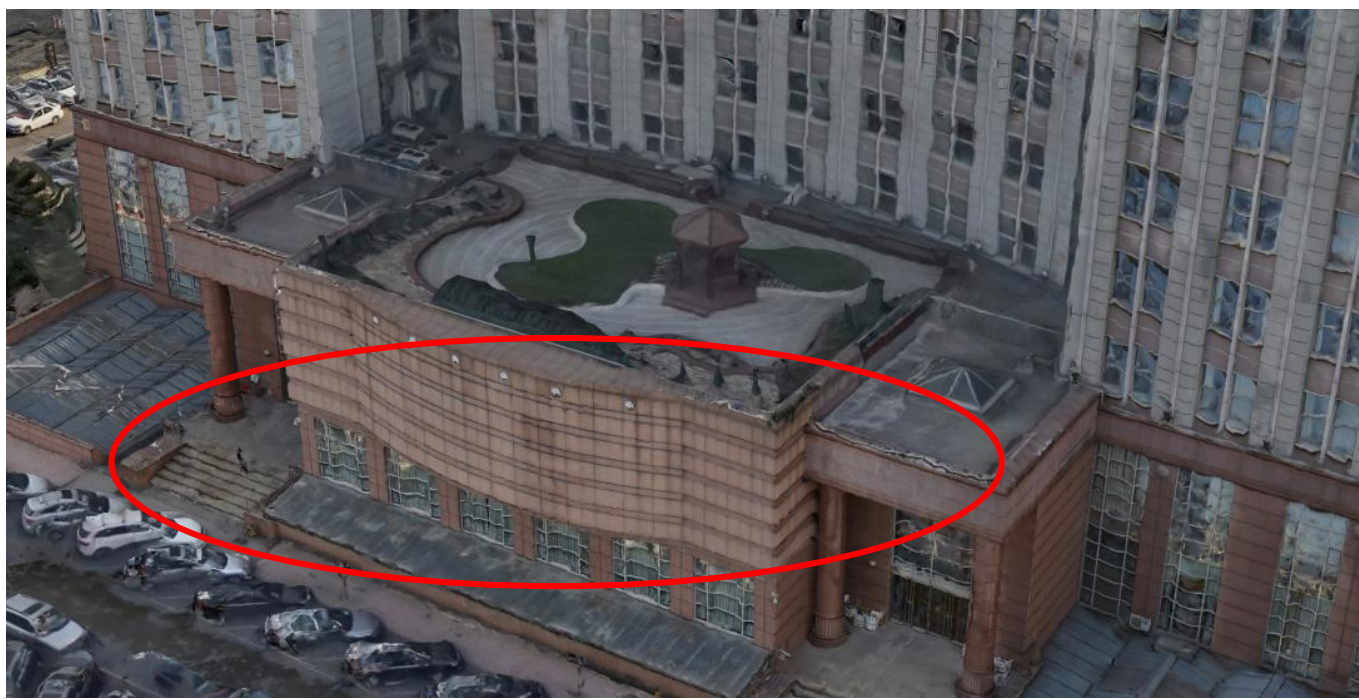

Figure 13 The air view of one big building model produced by OP and close range photogrammetry

According to the figure 11 to 13 , it also proved that the combination AT of the terrestrial and the oblique images can improve the model.

\section{CONCLUSIONS}

In this paper, to solve the problem of the partial deformation of the city real 3D models produced by the oblique photogrammetry, a new method by combining the oblique photogrammetry and the terrestrial photogrammetry was proposed, and One new terrestrial images acquisition device was also set up, which consists of six cameras and one GPS antenna on the top of the device. This new device can be mounted on the vehicle to acquire images with some certain time or distance interval. So it improved the data acquisition efficiency and can meet the requirement of the terrestrial photogrammetry.

According to the designed process, with the help of Context Capture software, the aerial triangulation (AT) was carried out with the terrestrial images and the oblique images. Based on the AT result, the model was produced. The model result has improved the partial deformation. This new model can meet the requirement of the smart city better. It advised that the data acquire time was very vital, so the time between the oblique photogrammetry and the close range photogrammetry should as near as possible. However, the accuracy and the efficiency of this new proposed method should be explored in actual projects in the future. 


\section{REFERENCES}

Yang, G. D., \& Wang, M. S. (2016). The tilt photographic measuration technique and expectation. Geomatics \& Spatial Information Technology.

Lin, Q. U., Feng, Y., Zhi, L. M., \& Gao, W. H. (2015). Study on real $3 \mathrm{~d}$ modeling of photographic data based on uav. Geomatics \& Spatial Information Technology.

$\mathrm{Hu}$ wenqing. (2016). Study on the application of three-dimensional reconstruction of tilt Photogrammetry in urban planning. Kunming University of science and technology Qiu Cunxia etc. (2017). 3D model construction and model optimization of sloping image. Bulletin of Surveying and Mapping.
Zhou Xiaomin etc. (2016)The construction method of urban real 3D model with tilt Photogrammetry. Science of Surveying and Mapping.

Wang Jianqiang etc. (2014). Urban 3D modeling method based on multi view aerial images. Science of Surveying and Mapping.

Context Capture User Manual version 4.4.(2017)

Revised April 2018 